Abanico Agroforestal. Enero-Diciembre 2020; 2:1-13. http://dx.doi.org/10.37114/abaagrof/2020.7 Artículo Original. Recibido: 17/02/2020. Aceptado: 10/07/2020. Publicado: 14/08/2020.

\title{
Extractos vegetales para el control de Fusarium oxysporum, Fusarium solani y Rhizoctonia solani, una alternativa sostenible para la agricultura
}

\author{
Plant extracts to control Fusarium oxisporum, Fusarium solani y Rhizoctonia solani: a \\ sustainable alternative for agriculture
}

\section{Alfredo Rodríguez-Castro ${ }^{1 \mathrm{ID}}$, Sandra Torres-Herrera ${ }^{2}$ ID , Antonio Domínguez- Calleros $^{2}$ ID , Ana Romero-García ${ }^{3}$ ID, Miguel Silva-Flores ${ }^{\star 1}$ ID}

${ }^{1}$ Tecnológico Nacional de México/Instituto Tecnológico Superior de Rioverde. Carretera Rioverde-San Ciro

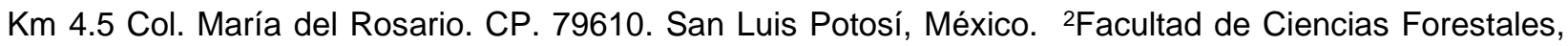
Universidad Juárez Estado de Durango. México. ${ }^{3}$ Instituto Potosino de Investigación Científica y Tecnológica A.C., Camino a La Presa de San José 2055, Lomas 4 sección. CP. 78216. San Luis Potosí, México. "Autor para correspondencia: Miguel Silva-Flores. ing.josealfredorodriguez@gmail.com, sith.chany@gmail.com, pdomingc@hotmail.com,

alrg_6@hotmail.com, miguelangelsilvaflores@gmail.com.

\section{RESUMEN}

Actualmente la agricultura requiere alternativas al uso de agrotóxicos para controlar fitopatógenos, los extractos vegetales pueden contribuir a minimizar pérdidas por fitopatógenos sin causar daños a la salud humana. El objetivo de este trabajo fue evaluar in vitro, el efecto de extractos de plantas sobre Fusarium oxysporum, Fusarium solani y Rhizoctonia solani. Se evaluaron los extractos metanólicos (EM) de: Moringa oleifera (Moringa), Persea americana (Aguacate), Equisetum hymale (Cola de caballo), Larrea tridentata (Gobernadora), Gnaphalium semiamplexicaule (Gordolobo), Peumus boldus (Boldo), Brickellia squarrosa (Prodigiosa), Rosmarinus officinalis (Romero) y Physalis coztomatl (Costomate), que se obtuvieron utilizando un equipo Soxhlet a una concentración del 10\% (p/V). Mediante el software estadístico Mlnitab $16{ }^{\circledR}$ México, se hizo un análisis de varianza (ANDEVA) y comparación de medias de Tukey $(p \leq 0.05)$. Por separado se determinó el porcentaje de inhibición del crecimiento micelial. El EM de Larrea tridentata (Gobernadora) inhibió al 100\% el crecimiento de Fusarium solani y de Rhizoctonia solani hasta por 144 h, y de F. oxysporum hasta por $240 \mathrm{~h}$. Los EM de Brickellia squarrosa (Prodigiosa) y Rosmarinus officinalis (Romero) también inhibieron el crecimiento micelial. Estos extractos representan una excelente alternativa al control y manejo convencional de fitopatógenos.

Palabras clave: fitopatógenos, Fusarium oxysporum, F. solani, Rhizoctonia solani, extractos vegetales y biocontrol.

ABSTRACT
Agriculture currently requires alternatives to the use of pesticides to control plant pathogens, such as plant
extracts that can help minimize losses from plant pathogens, without causing harm to human health. In this
work, the effect of plant extracts on Fusarium oxysporum, Fusarium solani and Rhizoctonia solani was
evaluated in vitro. The methanolic extracts (EM) of: Moringa oleifera (Moringa, leaves), Persea americana
(Avocado), Equisetum hymale (Horsetail), Larrea tridentata (Governor), Gnaphalium semiamplexicaule
(Gordolobo), Peumus boldus (Boldo), Brickellia squarrosa (Prodigiosa), Rosmarinus officinalis (Rosemary)
and Physalis coztomatl (Costomate), were obtained using a Soxhlet kit at a concentration of $10 \%$ (w/V).
Using the statistical software Mlnitab $16 \AA$ México, an analysis of variance (ANDEVA) and comparison of
Tukey means $(\mathrm{p} \leq 0.05$ ) were performed. The percentage of inhibition of mycelial growth was determined


separately. The ME of Larrea tridentata (Gobernadora) 100\% inhibited the growth of Fusarium solani and Rhizoctonia solani for up to $144 \mathrm{~h}$, and of $F$. oxysporum for up to $240 \mathrm{~h}$. The ME of Brickellia squarrosa (Prodigiosa) and Rosmarinus officinalis (Rosemary) also inhibited mycelial growth. These extracts represent an excellent alternative to the conventional control and management of plant pathogens.

Keywords: phytopathogens, Fusarium oxysporum, F. solani, Rhizoctonia solani, plant extracts and biocontrol.

\section{INTRODUCCIÓN}

Los avances científicos y tecnológicos consiguen aumentar la productividad de la agricultura. En general, el aumento se debe a la incorporación de productos sintéticos como fertilizantes y plaguicidas que generan problemas ambientales (Samsidar et al., 2018). Actualmente la agricultura requiere alternativas ecológicas, económicas y amigables con el ambiente para manejar enfermedades y minimizar el uso de agroquímicos sintéticos (Tamilselvi y Arumugam, 2017), que no perjudiquen la salud de los jornaleros agrícolas y consumidores (Samsidar et al., 2018).

Las enfermedades en la raíz en los cultivos son de los problemas más difíciles de controlar, porque en el suelo existen condiciones muy particulares que le brindan a los fitopatógenos de raíz (FR), elementos y condiciones óptimas para su establecimiento y desarrollo (García, 2010). Ante la necesidad de contar con soluciones ecológicas y de reducir el impacto negativo de los agrotóxicos en los ecosistemas, se ha encontrado en los extractos de plantas una opción sustentable para mitigar los problemas fitosanitarios y disminuir las pérdidas económicas que estos originan (Cerqueira et al., 2016).

Tradicionalmente el control y manejo de los problemas fitosanitarios se hace con agrotóxicos, este manejo acarrea consecuencias adversas en la salud y en el ecosistema; además de generar resistencia en los fitopatógenos a algunos compuestos de síntesis química empleados en la agricultura (Del Puerto et al., 2014). Sin embargo, se debe explorar la factibilidad del control de fitopatógenos con otras opciones como los extractos vegetales, los cuales pueden ser igual de efectivos para el control de y manejo de agentes fitopatógenos. Los extractos se pueden obtener a través de diferentes métodos: destilación con vapor-extracción con solvente, extracción con fluido supercrítico (Stashenko et al., 2003); soxhlet y lixiviación o percolación en frío (Henao et al., 2009).

Existen trabajos de investigación donde se emplean extractos de plantas para el manejo de agentes fitopatógenos, en ese sentido están los que se han hecho para; inhibición de Phytophthora infestans (Gamboa-Alvarado et al., 2003), control de Pythium (Lira-Saldívar et al., 2003; Osorio et al., 2010), control de Verticillium dahliae (López-Benítez et al., 2005), Sclerotinia sclerotiorum (Al-Reza et al., 2010), Fusarium oxysporum (Rodríguez et al., 2012; Cáceres Rueda de León et al., 2013; Vásquez et al., 2014; Dania et al., 2014; Ferdes et al., 2017), control de Fusarium solani (Zaker, 2014; Vásquez et al., 2014) y Rhizoctonia solani (López-Benítez et al., 2005; Jasso de Rodríguez et al., 2007; ZamoraNatera et al., 2008; Dania et al., 2014). 
El efecto de los extractos de las plantas sobre algunos patógenos, ya sean de interés médico o agrícola, se debe a que contienen metabolitos secundarios con efecto fungicida y/o bactericida, destacan los compuestos fenólicos, cumarinas, flavonoides, taninos, quinonas, entre otros. Existen trabajos donde se evalúa el efecto de plantas sobre diversos patógenos; por ejemplo, el efecto de los extractos de: Moringa oleífera (CanettRomero et al., 2014), Equisetum hymale (De Queiroz et al., 2015), Larrea tridentata (Bañuelos-Valenzuela et al., 2018), Peumus boldus (Mazutti et al., 2008) y Rosmarinus officinalis (Rozman y Jersek, 2009).

Por lo anterior, el objetivo del presente trabajo fue evaluar in vitro, el efecto de nueve extractos metanólicos, sobre los agentes fitopatógenos, Fusarium oxysporum, F. solani y Rhizoctonia solani. Con la hipótesis que los extractos vegetales son capaces de controlar e inhibir el crecimiento de algunos microorganismos fitopatógenos.

\section{MATERIAL Y MÉTODOS}

El estudio se realizó en las instalaciones del Instituto Tecnológico Superior de Rioverde (ITSR), ubicado en la Carretera Rioverde- San Ciro de Acosta, Km. 4.5, Col. María del Rosario, Rioverde, San Luis Potosí, México. Para el presente estudio se utilizaron extractos de nueve plantas reconocidas; las cuales fueron: Moringa oleifera (Moringa, hojas), Persea americana (Aguacate, hojas), Equisetum hymale (Cola de caballo, vástagos), Larrea tridentata (Gobernadora, hojas), Gnaphalium semiamplexicaule (Gordolobo, vástagos), Peumus boldus (Boldo, vástagos), Brickellia squarrosa (Prodigiosa, vástagos), Rosmarinus officinalis (Romero, vástagos) y Physalis coztomatl (Costomate, raíces).

Los extractos al 10\% p/V (peso/Volumen), se obtuvieron con el equipo Soxhlet (CornigPyrex Modelo $3840-\mathrm{XL}^{\circledR}$ ), durante cinco ciclos utilizando metanol (Merck $\left.{ }^{\circledR}\right)$ como solvente de arrastre. Los extractos se envasaron en frascos de vidrio ámbar y se almacenaron a $4^{\circ} \mathrm{C}$; la extracción con Soxhlet se hizo ocho días antes de la preparación de las cajas (Gamboa-Alvarado et al., 2003)

Los hongos fitopatógenos utilizados en este trabajo fueron Fusarium oxysporum, F. solani y Rhizoctonia solani. Estos microorganismos fueron aislados de cultivos locales de Jitomate (Solanum lycopersicum), e identificados mediante técnicas moleculares. La extracción del DNA se hizo siguiendo el protocolo descrito por Reader y Broda (1989). Se amplificó la secuencia de la región interna del transcrito del 18S Rdna, usando los oligos universales ITS1 (5'TCC GTA GGT GAA CCT GCG G 3') y el ITS4 (5'TCC TCC GCT TAT TGA TAT GC 3'), diseñados por White et al., (1990). Los productos del PCR fueron clonados en el vector pGEM-Teasy, siguiendo las instrucciones del fabricante (Promega 2015); se secuenciaron por el método de Sanger en el Laboratorio Nacional de Biotecnología Agrícola, Médica y Ambiental del Instituto Potosino de Investigación Científica y Tecnológica A.C. Una vez secuenciados los ITS se analizaron con BLAST 
(Basic Local Alignment Search Tool) (Morgulis et al., 2008) en el NCBI (National Center for Biotechnology Information) http://www.ncbi.nlm.nih.gov/BLAST/.

\section{Evaluación in vitro de los extractos vegetales contra fitopatógenos}

La evaluación del efecto de los extractos (25 ppm) se hizo mediante el método de cultivo envenenado (Grover,1962; Gamboa-Alvarado et al., 2003). Esta técnica consiste en incorporar el extracto vegetal en medio de cultivo Papa Dextrosa Agar (PDA BD Bioxon ${ }^{\circledR}$ México) y medir el crecimiento micelial de los fitopatógenos $F$. solani, F. oxysporum y $R$. solani. Los tratamientos de los extractos de moringa, gobernadora, aguacate, cola caballo, boldo, gordolobo, prodigiosa, romero y costomate, se evaluaron colocando 500 $\mu \mathrm{L}$ (25ppm) de los extractos por caja Petri $(20 \mathrm{~mL})$; se incluyó como control agar sin extracto alguno (solo PDA). Se evaluó por triplicado cada uno de los extractos contra cada uno de los fitopatógenos ( $R$. solani, $F$. solani y $F$. oxysporum), midiendo cada $24 \mathrm{~h}$ el crecimiento micelial con un vernier digital de precisión +/-.001", +/-.02 mm (Mitutoyo; 500-196-30C ${ }^{\circledR}$ ).

Para cultivar los microorganismos se utilizó medio PDA, $39 \mathrm{~g}$ por litro de agua destilada estéril. En cajas Petri de $90 \mathrm{~mm}$ de diámetro se vertieron $20 \mathrm{~mL}$ de una mezcla PDA:EM (Papa Dextrosa Agar: Extracto Metanólico), en una proporción de 1:0.05; es decir, 500 $\mu \mathrm{L}$ de extracto por caja. Todo lo anterior en campana de flujo laminar (Marca

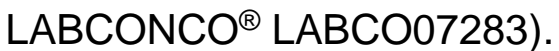

Después de este tiempo las cajas se inocularon con un explante de $5 \mathrm{~mm}$ de diámetro de PDA, con crecimiento del fitopatógeno y se incubaron en una cámara bioclimática a $25^{\circ} \mathrm{C}$ (Marca Thermo Scientific ${ }^{\circledR}$ Mod 3949). Todas las pruebas se hicieron por triplicado en un diseño experimental completamente al azar.

\section{Análisis de los datos}

Con los datos obtenidos en la fase experimental se calculó el porcentaje de inhibición del crecimiento micelial con la fórmula:

$$
\text { I. C. M. }=[(\mathrm{Dc}-\mathrm{Dt}) / \mathrm{Dc}] x 100
$$

dónde: I.C.M. es el porcentaje de inhibición del crecimiento del micelio, Dc es el diámetro del micelio en el control y $\mathrm{Dt}=$ diámetro del micelio en el tratamiento.

Asimismo, se hizo el análisis de varianza (ANDEVA) y la comparación de medias múltiple de Tukey $(p \leq 0.05)$. El análisis se hizo con el software estadístico Minitab $16^{\circledR}$ México.

\section{RESULTADOS Y DISCUSIÓN}

Al evaluar el efecto de los Extractos Metanólicos (EM) sobre Fusarium oxysporum, se observó diferencia estadística entre los tratamientos ( $p \leq 0.05)$. El EM de L. tridentata inhibió al $100 \%$ el crecimiento del patógeno hasta por 144 h, y $90 \%$ hasta por 240 h; mientras que el de $R$. officinalis inhibió el $50.7 \%$ hasta 72 h, y $42.2 \%$ por 144 h (cuadro 
1). López-Benítez et al., en el 2005, presentaron resultados similares, en los que indican que los extractos de Syzygium aromaticum y L. tridentata al $10 \%$ inhiben el crecimiento de F. oxysporum f. sp. Lycopersici, hasta por 144 h. Dania et al., en el 2014, documentaron que los extractos acuosos (EA) de Oryza sativa y Quercus phillyraeoides al 1.5 y $2.5 \%$ pueden inhibir in vitro totalmente el crecimiento de seis fitopatógenos, entre ellos F. oxysporum.

Por otra parte los extractos etanólicos (EE) de Fluorensia cernua (Hojasén), $F$. microphylla y $F$. retinophylla mostraron ser una alternativa efectiva para el control de Fusarium oxysporum, inhibiendo el $100 \%$ su crecimiento, con una concentración de 1500 $\mu \mathrm{L} \mathrm{L}^{-1}$ (Jasso de Rodríguez et al., 2007).

Existen trabajos donde también se han evaluado Aceites Esenciales (AE) para el manejo de F. oxysporum; en ese sentido está el trabajo de Ferdes et al., en el 2017, con AE de $R$. officinalis, en una concentración de $20 \mu \mathrm{g} \mathrm{mL}^{-1}$ se inhibe el $93 \%$ del crecimiento de $F$. oxysporum; mientras que con $10 \mu \mathrm{g} \mathrm{mL}^{-1}$ de los AE de $P$. anisum y $S$. hortensis se inhibe totalmente el crecimiento de F. oxysporum. Asimismo Vásquez et al., en el 2014, concluyeron que los AE de Chenopodium ambrosioides al $2 \%$ y Chenopodium album al $0.03 \%$, inhiben por completo el crecimiento de F. oxysporum f. sp. Lycopersici, hasta por 11 días.

Cuadro 1. Inhibición del crecimiento micelial (ICM, \%) de Fusarium oxysporum con diferentes extractos metanólicos a 72, 144 y $240 \mathrm{~h}$, después del inicio del experimento

\begin{tabular}{|c|c|c|c|c|c|c|}
\hline $\begin{array}{l}\text { Extracto } \\
\text { vegetal }\end{array}$ & $72 \mathrm{~h}$ & & $144 \mathrm{~h}$ & & $240 \mathrm{~h}$ & \\
\hline L. tridentata & $100.0 \pm 0.0$ & a & $100.0 \pm 0.0$ & a & $89.4 \pm 0.3$ & $\mathrm{a}$ \\
\hline B. squarrosa & $39.8 \pm 4.4$ & $\mathrm{bc}$ & $38.6 \pm 2.3$ & $\mathrm{bc}$ & $31.7 \pm 2.7$ & $b$ \\
\hline G. semiamplexicaule & $24.6 \pm 2.3$ & $\mathrm{~cd}$ & $28.3 \pm 1.2$ & $c d$ & $24.2 \pm 2.4$ & $\mathrm{bc}$ \\
\hline$P$. americana & $25.1 \pm 5.4$ & $c d$ & $26.3 \pm 2.2$ & $d$ & $12.9 \pm 4.9$ & $\mathrm{~cd}$ \\
\hline$P$. boldus & $26.0 \pm 3.9$ & $\mathrm{~cd}$ & $24.5 \pm 4.1$ & $d$ & $11.7 \pm 2.7$ & $\mathrm{~cd}$ \\
\hline P. coztomatl & $24.8 \pm 4.2$ & $\mathrm{~cd}$ & $27.4 \pm 1.5$ & $\mathrm{~cd}$ & $9.2 \pm 4.0$ & $\mathrm{bc}$ \\
\hline M. oleifera & $19.7 \pm 3.4$ & $d$ & $17.1 \pm 2.9$ & $d$ & $4.0 \pm 1.4$ & d \\
\hline R. officinalis & $50.7 \pm 2.9$ & $\mathrm{~b}$ & $42.2 \pm 1.5$ & $b$ & $29.6 \pm 2.2$ & $\mathrm{~b}$ \\
\hline
\end{tabular}

Medias con letra diferente indican diferencia estadística (Tukey, $p \leq 0.05$ ).

En este estudio, el EM de L. tridentata inhibió el crecimiento de F. oxysporum, hasta por $144 \mathrm{~h}$, seguido en efectividad por el de $R$. officinalis y $B$. squarrosa. Con estos tratamientos se inhibe el crecimiento micelial hasta $80 \%$, comparado con el control negativo (cuadro 2). Estos resultados coinciden con los reportados por Osorio et al., en el 2010, ellos concluyen que con una concentración de $0.7 \mathrm{mg} \mathrm{kg}^{-1} \mathrm{~L}$. tridentata inhibe el $100 \%$ el crecimiento de F. oxisporum in vitro.

En los resultados de los extractos sobre Fusarium solani, mediante el ANDEVA y la comparación de medias Tukey $(p \leq 0.05)$, se observó diferencia estadística significativa entre los tratamientos. EI EM de L. tridentata inhibió el $100 \%$ del crecimiento de F. solani, hasta por diez días; mostrando un efecto de inhibición importante sobre este fitopatógeno. 
Lo anterior es relevante si se considera que hay productos químicos que se aplican una o más veces por semana y no logran estos resultados, incluso en condiciones de laboratorio no logran inhibir al $100 \%$ el crecimiento del micelio, tal como lo reportan Yossen y Conles en el 2016, que en su trabajo con moléculas comerciales, alcanzan una inhibición de entre el 60 y el 97\%. El EM de $R$. officinalis evaluado en este trabajo inhibió en un $70 \%$ el crecimiento del micelio, respecto al control hasta por $144 \mathrm{~h}$. Este dato es similar al obtenido con extractos acuosos de Prosopis juliflora y Lantana camara, que consiguen un 80 y 69\% de inhibición micelial, respectivamente (Seetha et al., 2010). Asimismo, los estudios de David et al., en el 2013, reportan que el EM de botones florales de Calotropis gigantea al $25 \%$, reduce $68 \%$ el crecimiento de F. solani.

Cuadro 2. Crecimiento micelial promedio $(\mathrm{mm})$ de Fusarium oxysporum con diferentes extractos metanólicos a 72, 144 y $240 \mathrm{~h}$, después del inicio del experimento

\begin{tabular}{|c|c|c|c|c|c|c|}
\hline Extracto vegetal & \multicolumn{2}{|l|}{$72 \mathrm{~h}$} & \multicolumn{2}{|l|}{$144 \mathrm{~h}$} & \multicolumn{2}{|c|}{$240 \mathrm{~h}$} \\
\hline Agar & $22.3 \pm 0.4$ & $\mathrm{a}$ & $44.4 \pm 0.7$ & $\mathrm{a}$ & $65.9 \pm 0.7$ & $a$ \\
\hline L. tridentata & $0.0 \pm 0.0$ & e & $0.0 \pm 0.0$ & e & $7.0 \pm 0.2$ & e \\
\hline B. squarrosa & $13.4 \pm 1.0$ & $\mathrm{~cd}$ & $27.3 \pm 1.0$ & $\mathrm{~cd}$ & $45.0 \pm 1.7$ & $d$ \\
\hline R. officinalis & $11.0 \pm 0.6$ & $d$ & $25.7 \pm 0.6$ & $d$ & $46.4 \pm 1.4$ & $d$ \\
\hline G. semiamplexicaule & $16.8 \pm 0.5$ & $\mathrm{bc}$ & $31.8 \pm 0.5$ & bc & $50.0 \pm 1.6$ & $c d$ \\
\hline P. coztomatl & $16.8 \pm 0.9$ & $\mathrm{bc}$ & $32.3 \pm 0.7$ & bc & $53.3 \pm 2.6$ & $c d$ \\
\hline E. hymale & $18.2 \pm 0.1$ & $\mathrm{~b}$ & $35.4 \pm 1.5$ & $b$ & $56.7 \pm 1.7$ & $\mathrm{bc}$ \\
\hline P. americana & $16.7 \pm 1.2$ & $\mathrm{bc}$ & $32.7 \pm 1.0$ & $b$ & $57.5 \pm 3.2$ & $a b c$ \\
\hline P. boldus & $16.5 \pm 0.9$ & $\mathrm{bc}$ & $33.5 \pm 1.8$ & $b$ & $58.3 \pm 1.8$ & $a b c$ \\
\hline M. oleifera & $17.9 \pm 0.8$ & $\mathrm{~b}$ & $36.8 \pm 1.3$ & $b$ & $63.3 \pm 0.9$ & $a b$ \\
\hline
\end{tabular}

Medias con letra diferente indican diferencia estadística (Tukey, $p \leq 0.05$ )

Cuadro 3. Inhibición del crecimiento micelial (ICM, \%) de Fusarium solani con diferentes extractos metanólicos a 72, 144 y $240 \mathrm{~h}$, después del inicio del experimento

\begin{tabular}{lrlrlrl}
\hline \multicolumn{1}{c}{ Extracto vegetal } & $72 \mathrm{~h}$ & $44 \mathrm{~h}$ & $240 \mathrm{~h}$ \\
\hline L. tridentata & $100.0 \pm 0.0$ & $\mathrm{a}$ & $100.0 \pm 0.0$ & $\mathrm{a}$ & $100.0 \pm 0.0$ & $\mathrm{a}$ \\
R. officinalis & $64.7 \pm 0.9$ & $\mathrm{~b}$ & $70.2 \pm 0.3$ & $\mathrm{~b}$ & $58.5 \pm 1.7$ & $\mathrm{~b}$ \\
B. squarrosa & $37.9 \pm 0.4$ & $\mathrm{c}$ & $37.2 \pm 1.3$ & $\mathrm{c}$ & $11.8 \pm 0.9$ & $\mathrm{c}$ \\
P. coztomatl & $26.5 \pm 1.2 \mathrm{de}$ & $28.5 \pm 1.8 \mathrm{~d}$ & $5.7 \pm 0.4$ & $\mathrm{~d}$ \\
P. americana & $26.9 \pm 2.5 \mathrm{de}$ & $28.4 \pm 1.2 \mathrm{~d}$ & $0.5 \pm 0.5$ & $\mathrm{e}$ \\
P. boldus & $31.2 \pm 1.2 \mathrm{~cd}$ & $30.8 \pm 0.3 \mathrm{~d}$ & $0.0 \pm 0.0$ & $\mathrm{e}$ \\
G. semiamplexicaule & $26.3 \pm 2.0 \mathrm{de}$ & $28.1 \pm 0.3 \mathrm{~d}$ & $0.0 \pm 0.0$ & $\mathrm{e}$ \\
M. oleifera & $22.6 \pm 3.4 \mathrm{de}$ & $15.5 \pm 0.9$ & $\mathrm{f}$ & $0.0 \pm 0.0$ & $\mathrm{e}$ \\
E. hymale & $21.5 \pm 2.4$ e & $22.2 \pm 0.6 \mathrm{e}$ & $0.0 \pm 0.0$ & $\mathrm{e}$ \\
\hline
\end{tabular}

Medias con letra diferente indican diferencia estadística (Tukey, $p \leq 0.05$ )

Es importante contar con opciones ecológicas para el control de hongos fitopatógenos, como las exploradas en este trabajo. En esta investigación, los resultados con el EM de L. tridentata coincidieron con los de Osorio et al., en el 2010, quienes utilizaron extractos polifenólicos de L. tridentata, y consiguen inhibir el $100 \%$ del crecimiento de F. solani, con una concentración de $0.70 \mathrm{mg} \mathrm{L}^{-1}$. 
En un estudio conducido por Vásquez et al., (2014), se evaluó el efecto fungistático de extractos acuosos (EA) y aceites esenciales ( $\mathrm{AE}$ ) de especies del género Chenopodium sobre F. solani; los autores concluyeron que el AE de Chenopodium ambrosioides al $2 \%$ y Chenopodium album al $0.03 \%$ inhiben por completo el crecimiento de $F$. solani, hasta por 11 días; mientras que en este trabajo L. tridentata lo inhibió al 100\% hasta por 10 días. Otro caso con resultados similares sobre Fusarium spp. fue el trabajo de Duarte et al., en el 2013, quienes con aceites esenciales de Piper aduncum subsp. ossanum y Piper aurintum inhiben totalmente el crecimiento de este hongo.

Por otra parte, Zaker (2014) concluyó que el EM de hojas de Artemisia annua al 15\%, es capaz de inhibir el crecimiento de $F$. solani; mientras que en el presente trabajo en EM de $L$. tridentata inhibió al $100 \%$ el crecimiento de $F$. solani, seguido de los extractos de $R$. officinalis y $B$. squarrosa; los cuales, aunque en menor proporción también lo inhiben (cuadro 4).

Cuadro 4. Crecimiento micelial promedio $(\mathrm{mm})$ de Fusarium solani, con diferentes extractos metanólicos a 72, 144 y 240 h, después del inicio del experimento

\begin{tabular}{lrrrrr}
\hline \multicolumn{1}{c}{ Extracto vegetal } & $72 \mathrm{~h}$ & $144 \mathrm{~h}$ & $240 \mathrm{~h}$ \\
\hline Agar & $30.1 \pm 0.4$ & $\mathrm{a}$ & $66.6 \pm 0.7$ & $\mathrm{a}$ & $80.0 \pm 0.0 \mathrm{a}$ \\
L. tridentata & $0.0 \pm 0.0$ & $\mathrm{f}$ & $0.0 \pm 0.0$ & $\mathrm{~g}$ & $0.0 \pm 0.0 \mathrm{e}$ \\
R. officinalis & $10.6 \pm 0.3$ & $\mathrm{e}$ & $19.8 \pm 0.2$ & $\mathrm{f}$ & $33.2 \pm 1.3 \mathrm{~d}$ \\
B. squarrosa & $18.7 \pm 0.1$ & $\mathrm{~d}$ & $41.9 \pm 0.9$ & $\mathrm{e}$ & $70.5 \pm 0.8 \mathrm{c}$ \\
P. coztomatl & $22.1 \pm 0.3 \mathrm{bc}$ & $47.6 \pm 1.2$ & $\mathrm{~d}$ & $75.5 \pm 0.3 \mathrm{~b}$ \\
P. americana & $22.0 \pm 0.8 \mathrm{bc}$ & $47.7 \pm 0.8$ & $\mathrm{~d}$ & $79.6 \pm 0.4 \mathrm{a}$ \\
P. boldus & $20.7 \pm 0.4 \mathrm{~cd}$ & $46.1 \pm 0.2$ & $\mathrm{~d}$ & $80.0 \pm 0.0 \mathrm{a}$ \\
G. semiamplexicaule & $22.2 \pm 0.6 \mathrm{bc}$ & $47.9 \pm 0.2$ & $\mathrm{~d}$ & $80.0 \pm 0.0 \mathrm{a}$ \\
M. oleifera & $23.3 \pm 1.0 \mathrm{bc}$ & $56.3 \pm 0.6$ & $\mathrm{~b}$ & $80.0 \pm 0.0 \mathrm{a}$ \\
E. hymale & $23.7 \pm 0.7$ & $\mathrm{~b}$ & $51.8 \pm 0.4$ & $\mathrm{c}$ & $80.0 \pm 0.0 \mathrm{a}$ \\
\hline
\end{tabular}

Medias con letra diferente indican diferencia estadística (Tukey, $p \leq 0.05$ )

El extracto metanólico (EM) de L. tridentata, inhibe hasta el $100 \%$ del crecimiento de Rhizoctonia solani, durante los primeros diez días. Lo anterior indica que este extracto es un fungistático eficaz. Con el ANDEVA y la comparación de medias de Tukey con una significancia del $95 \%$ se observó diferencia estadística significativa entre tratamientos, ya que además de $L$. tridentata, el EM de $R$. officinalis también presenta un porcentaje de inhibición destacable, causa un efecto fungistático del 56 y $48 \%$ a las 144 h y $240 \mathrm{~h}$, respectivamente (cuadro 5).

Controlar el crecimiento de hongos fitopatógenos con extractos vegetales, representa un gran avance en materia de protección vegetal, en el caso de Rhizoctonia solani; GamboaAlvarado et al., en el 2003 reportaron resultados similares a los obtenidos en este trabajo de investigación; encontraron que el EM de Fluorensia cernua (Hojasén) a una concentración de $20000 \mathrm{mg} \mathrm{L}^{-1}$, inhibe $85 \%$ el crecimiento de este hongo hasta por 96 horas, respecto al control. López-Benítez et al., en el 2005, con extractos acuosos de $L$. tridentata y Cinnamomum zeylanicum al $10 \%$; de Syzygium aromaticum al $5 \%$, lograron 
inhibir in vitro el crecimiento hasta por $144 \mathrm{~h}$, y con el extracto acuoso de Quercus phillyraeoides al 3.5\% inhibieron en $94 \%$ el crecimiento del fitopatógeno. De igual manera el extracto alcaloideo de Lupinus mexicanus contra $R$. solani en una concentración de 5 $\mathrm{mg} \mathrm{mL}^{-1}$ inhibe al $100 \%$ su crecimiento (Zamora-Natera et al., 2008). Jasso de Rodríguez et al., (2007) mencionan que los extractos etanólicos de Flourensia cernua y $F$. retinophylla a una concentración de $1000 \mu \mathrm{L} \mathrm{L}^{-1}$ inhiben su crecimiento; de igual manera Al-Reza et al., en el 2010, determinaron que el AE de Cestrum nocturnum tiene un alto poder fungistático capaz de inhibir hasta $80 \%$ el crecimiento de $R$. solani; también Touba et al., en el 2012, encontraron que el EA de Kaempferia galanga puede inhibirlo totalmente. Por otra parte, Dania et al., en el 2014, demuestran que el extracto acuoso de Oryza sativa Husk al 1\%, in vitro, inhibe totalmente el crecimiento de este fitopatógeno.

Cuadro 5. Inhibición del crecimiento micelial (ICM, \%) en el tiempo de Rhizoctonia solani con diferentes extractos metanólicos a 72, 144 y $240 \mathrm{~h}$, después del inicio del experimento

\begin{tabular}{lrlrlrl}
\hline \multicolumn{1}{c}{ Extracto vegetal } & $72 \mathrm{~h}$ & $144 \mathrm{~h}$ & $240 \mathrm{~h}$ & \\
\hline L. tridentata & $100.0 \pm 0.0$ & $\mathrm{a}$ & $100.0 \pm 0.0$ & $\mathrm{a}$ & $100.0 \pm 0.0$ & $\mathrm{a}$ \\
R. officinalis & $56.8 \pm 0.9$ & $\mathrm{~b}$ & $56.2 \pm 1.9$ & $\mathrm{~b}$ & $48.0 \pm 3.0$ & $\mathrm{~b}$ \\
B. squarrosa & $32.7 \pm 1.7$ & $\mathrm{c}$ & $23.2 \pm 2.2$ & $\mathrm{c}$ & $17.8 \pm 2.2$ & $\mathrm{c}$ \\
P. americana & $24.5 \pm 1.6$ & $\mathrm{c}$ & $19.1 \pm 1.2$ & $\mathrm{~cd}$ & $8.1 \pm 1.3$ & $\mathrm{~d}$ \\
P. coztomatl & $26.9 \pm 3.9$ & $\mathrm{c}$ & $21.6 \pm 1.4$ & $\mathrm{~cd}$ & $7.5 \pm 1.1$ & $\mathrm{~d}$ \\
P. boldus & $28.7 \pm 2.2$ & $\mathrm{c}$ & $21.3 \pm 1.1$ & $\mathrm{~cd}$ & $7.5 \pm 2.3$ & $\mathrm{~d}$ \\
E. hymale & $25.9 \pm 1.9$ & $\mathrm{c}$ & $18.5 \pm 0.2$ & $\mathrm{~cd}$ & $2.7 \pm 0.9$ & $\mathrm{~d}$ \\
M. oleifera & $24.4 \pm 3.1$ & $\mathrm{c}$ & $17.0 \pm 3.9$ & $\mathrm{~cd}$ & $1.8 \pm 0.9 \mathrm{~d}$ \\
G. semiamplexicaule & $23.8 \pm 0.7$ & $\mathrm{c}$ & $14.1 \pm 1.3 \mathrm{~d}$ & $1.1 \pm 0.5$ & $\mathrm{~d}$ \\
\hline
\end{tabular}

Medias con letra diferente indican diferencia estadística (Tukey, $p \leq 0.05$ )

Cuadro 6. Crecimiento micelial promedio $(\mathrm{mm})$ de Rhizoctonia solani, con diferentes extractos metanólicos a 72, 144 y $240 \mathrm{~h}$, después del inicio del experimento

\begin{tabular}{|c|c|c|c|c|c|c|}
\hline Extracto vegetal & \multicolumn{2}{|l|}{$72 \mathrm{~h}$} & \multicolumn{2}{|l|}{$144 \mathrm{~h}$} & \multicolumn{2}{|l|}{$240 \mathrm{~h}$} \\
\hline Agar & $19.9 \pm 0.2$ & a & $45.8 \pm 0.2$ & $\mathrm{a}$ & $80.0 \pm 0.0$ & a \\
\hline L. tridentata & $0.0 \pm 0.0$ & d & $0.0 \pm 0.0$ & e & $0.0 \pm 0.0$ & e \\
\hline R. officinalis & $9.6 \pm 0.2$ & c & $19.5 \pm 0.8$ & $d$ & $34.3 \pm 2.0$ & d \\
\hline B. squarrosa & $15 \pm 0.4$ & b & $34.1 \pm 1.0$ & c & $54.2 \pm 1.5$ & c \\
\hline E. hymale & $16.5 \pm 0.4$ & b & $36.2 \pm 0.1$ & bc & $64.2 \pm 0.6$ & b \\
\hline G. semiamplexicaule & $17 \pm 0.1$ & b & $38.1 \pm 0.6$ & b & $65.2 \pm 0.3$ & b \\
\hline M. oleifera & $16.9 \pm 0.7$ & b & $36.9 \pm 1.7$ & bc & $64.7 \pm 0.6$ & b \\
\hline P. americana & $16.8 \pm 0.3$ & b & $35.9 \pm 0.5$ & bc & $60.6 \pm 0.9$ & b \\
\hline P. boldus & $15.9 \pm 0.5$ & b & $34.9 \pm 0.5$ & bc & $61.0 \pm 1.5$ & b \\
\hline P. coztomatl & $16.3 \pm 0.9$ & b & $34.8 \pm 0.6$ & bc & $61.0 \pm 0.7$ & b \\
\hline
\end{tabular}

Medias con diferente letra indican diferencia estadística (Tukey, $p \leq 0.05$ ) 


\section{CONCLUSIÓN}

El extracto metanólico de gobernadora (Larrea tridentata), es efectivo para inhibir el crecimiento de Fusarium oxysporum, Fusarium solani y Rhizoctonia solani, hasta por diez días. De igual manera se concluye que el extracto metanólico de Rosamarinus officinalis (Romero), se puede usar para el manejo de Fusarium oxysporum, Fusarium solani y Rhizoctonia solani, con menor efectividad que el de gobernadora.

\section{Agradecimientos}

Al proyecto de Fondos Mixtos FOMIX-SLP (FMSLP-2013-C01-209337) por el financiamiento de este trabajo de investigación. Al Ing. Fernando Mendoza González y a la M.C. Sonia Castillo Gutiérrez, por su colaboración en la revisión del documento de informe de resultados. Al Centro para la Integración del Desarrollo Agroecológico y Sosteniblidad "El Humedal" en Valle de Bravo, estado de México, por las facilidades brindadas.

\section{LITERATURA CITADA}

AL-REZA S, Rahman A, Ahmed Y, Kang S. 2010. Inhibition of plant pathogens in vivo and in vitro with essential oil and organic extracts of Cestrum nocturnum L. Pestic. Biochem. Physiol. 96: 86-92. https://doi.org/10.1016/j.pestbp.2009.09.005

BAÑUELOS-VALENZUELA R, Delgadillo-Ruiz L, Echavarría-Cháirez F, Delgadillo-Ruiz O, Meza-López C. 2018. Composición química y FTIR de extractos etanólicos de Larrea tridentata, Origanum vulgare, Artemisa ludoviciana y Ruta graveolens. Agrociencia, 52(3), 309-321. http://www.colpos.mx/agrocien/Bimestral/2018/abr-may/art-2.pdf

CÁCERES RUEDA DE LEON I, Colorado R, Salas E, Muñoz L, Hernández L. 2013. Actividad Antifúngica in vitro de Extractos Acuosos de especias contra Fusarium oxysporum, Alternaria alternata, Geotrichum candidum, Trichoderma spp., Penicillum digitatum y Aspergillus niger. Rev. Mex. Fitopatol. 31:105-112. ISSN 0185-3309. http://www.scielo.org.mx/scielo.php?script=sci_arttext\&pid=S0185-33092013000200003

CANETT-ROMERO R, Arvayo-Mata KL, Ruvalcaba-Garfias NV. 2014. Aspectos tóxicos más relevantes de Moringa oleifera y sus posibles daños. Biotecnia, 16(2), 36-43. https://biotecnia.unison.mx/index.php/biotecnia/article/view/45/41

CERQUEIRA M, Barcellos H, Bueno P, Aires J, Dummer M. 2016. Antifungal activity of plants extracts with potential to control plant pathogens in pineapple. Asian Pac J Trop Biomed. 6:26-31. https://doi.org/10.1016/j.apjtb.2015.09.026

DANIA V, Fadnia O, Ayodele M, Kumar L. 2014. Efficacy of Oryza sativa husk and Quercus phillyraeoides extracts for the in vitro and in vivo control of fungal rot disease of white yam (Discorea rotundata Poir). SpingerPlus. 3:711. https://doi.org/10.1186/21931801-3-711 
DAVID M, Kumar R, Bhavani M. 2013. Experimental studies on active metabolites of Calotropis gigantea for evaluation of possible antifungal properties. Int. Res. J. Pharm. 4:250-254.

https://www.researchgate.net/publication/239524862_Experimental_Studies_on_active_ metabolites_of_Calotropis_gigantea_for_evaluation_of_possible_antifungal_properties

DE QUEIROZ GM, Politi FA, Rodrigues ER, Souza-Moreira TM, Moreira RR, Cardoso CR, Pietro RC. 2015. Phytochemical characterization, antimicrobial activity, and antioxidant potential of Equisetum hyemale L.(Equisetaceae) extracts. Journal of medicinal food, 18(7),
https:/www.liebertpub.com/doi/abs/10.1089/jmf2014.0089

DEL PUERTO RODRIGUEZ AM, Suárez Tamayo S, Palacio Estrada DE. 2014. Efectos de los plaguicidas sobre el ambiente y la salud. Revista Cubana de Higiene $y$ Epidemiología. 52(3): 372-387.

https://www.researchgate.net/publication/317518438_Efectos_de_los_plaguicidas_sobr e_el_ambiente_y_la_salud

DUARTE Y, Pino O, Martínez B. 2013. Efecto de cuatro aceites esenciales sobre Fusarium spp. Rev. Prot. Veg. 28:232-235.

http://scielo.sld.cu/scielo.php?script=sci_arttext\&pid=S1010-27522013000300013

FERDES M, Al Juhaimi F, Özcan M, Ghafoor K. 2017. Inhibitory effect of some plant essential oils on growth of Aspergillus niger, Aspergillus oryzae, Mucor pusillus and Fusarium oxysporum. S. Afr. J. Bot. 113: 457460.https://doi.org/10.1016/j.sajb.2017.09.020

GAMBOA-ALVARADO R, Hernández-Castillo F, Guerrero-Rodríguez E, Sánchez-Arizpe A. 2003. Inhibición del crecimiento Micelial de Rhizoctonia solani Kühn y Phytophthora infestans Mont. (De Bary) con extractos vegetales Metanólicos de Hojasén (Flourensia cernua D.C.), mejorana (Origanum majorana L.) y trompetilla [Bouvardia ternifolia (Ca.) Shlecht.]. Rev. Mex. Fitopatol. 21:13-18. https://www.redalyc.org/pdf/612/61221102.pdf

GARCÍA R. 2010. Agroecología y enfermedades de la raíz en cultivos agrícolas. Editorial COLPOS. México. Pp. 130. ISBN: 9786077699088.

GROVER RK, More JD. 1962. Toxicometric studies of fungicides against the brown rot organism Sclerotinia fructivola and Sclerotinia laxa. Phytopathology. 52:876-880. 
HENAO J, Muñoz LJ, Ríos E, Padilla L, Giraldo GA. 2009. Evaluación de la actividad antimicrobiana de los extractos de la planta Lippia origanoides HBK cultivada en el Departamento del Quindío. Rev Invest Univ Quindio, 19, 159-64. http://blade1.uniquindio.edu.co/uniquindio/revistainvestigaciones/adjuntos/pdf/f4d9_n19 18.pdf

JASSO DE RODRIGUEZ D, Hernández-Castillo D, Angulo-Sánchez JL, RodríguezGarcía R, Villarreal-Quintanilla JA, Lira-Saldívar RH. 2007. Antifungal activity in vitro of Flourensia spp. extracts on Alternaria sp., Rhizoctonia solani, and Fusarium oxysporum. Ind. Crop. Prod. 25(2):111-116. https://doi.org/10.1016/j.indcrop.2006.08.007

LIRA-SALDíVAR R, Balvantín-García G, Hernández-Castillo F, Gamboa-Alvarado R, Jasso-de-Rodríguez D, Jiménez-Díaz F. 2003. Evaluation of Resin Content and the Antifungal Effect of Larrea tridentata (Sesse and Moc. Ex D.C.) Coville Extracts from Two Mexican Deserts Aganist Phythium sp. Pringsh. Rev. Mex. Fitopatol. 21:97-101. https://www.redalyc.org/articulo.oa?id=61221201

LÓPEZ-BENÍTEZ A, López-Betancourt S, Vázquez-Badillo M, Rodríguez-Herrera S, Mendoza-Elos M, Padrón-Corral E. 2005. Inhibición del crecimiento micelial de Fusarium oxysporum schlechtend. F. sp. lycopersici (Sacc.) Snyder y Hansen, Rhizoctonia Solani Kühn y Verticullium dahliae kleb mediante extractos vegetales acuosos. Rev. Mex. Fitopatol. 23:183-190. https://www.redalyc.org/articulo.oa?id=61223212

MAZUTTI M, Mossi AJ, Cansian RL, Corazza ML, Dariva C, Oliveira JV. 2008. Chemical profile and antimicrobial activity of Boldo (Peumus boldus Molina) extracts obtained by compressed carbon dioxide extraction. Brazilian Journal of Chemical Engineering. 25(2), 427-434.

https://www.scielo.br/scielo.php?pid=S01046322008000200020\&script=sci_arttext

MORGULIS A, Coulourios G, Raytselis Y, Madden TL, Agarwala R, Schäffe AA. 2008. Database indexing for production MegaBLAST searches. Bioinformatics vol. 24, no 16, p. 1757-1764. https://academic.oup.com/bioinformatics/article/24/16/1757/202524

OSORIO E, Flores M, Hernández D, Ventura J, Rodríguez R, Aguilar C. 2010. Biological efficiency of polyphenolic extracts from pecan nuts Shell (Carya illinoensis), pomegranate husk (Pnica granatum) and creosote bush leaves (Larrea tridentata Cov.) against plant $\begin{array}{llll}\text { pathogenic fungi. Ind. Crop. Prod. } & \text { 31:153-157. }\end{array}$ https://doi.org/10.1016/j.indcrop.2009.09.017

PROMEGA 2015. Technical manual. pGEM®-T y pGEM®- T Easy Vector Systems. Instructions for use of products. https://worldwide.promega.com/products/pcr/pcrcloning/pgem-t-easy-vector-systems/?catNum=A1360 
RAEDER U, Broda P. 1985. Rapid preparation of DNA from filamentous fungi. Letters in Applied Microbiology. vol. 1, no 1, p. 17-20. https://doi.org/10.1111/j.1472765X.1985.tb01479.x

RODRÍGUEZ A, Ramírez M, Bautista S, Cruz A, Rivero D. 2012. Actividad antifúngica de extractos de Acacia Farnesiana sobre el crecimiento in vitro de Fusarium oxysporum f. sp. lycopersici. Revista Científica UDO Agrícola. 12:91-96. http://www.bioline.org.br/pdf?cg12011

ROZMAN T, Jersek B. 2009. Antimicrobial activity of rosemary extracts (Rosmarinus officinalis L.) against different species of Listeria. Acta agriculturae Slovenica, 93(1), 51. https://pdfs.semanticscholar.org/0ad5/a7c3ece5e98fa57738406ae2c819923cf3f0.pdf

SÁNCHEZ LG, Vargas-Rincón A, Jiménez P. 2015. Evaluación de la actividad antifúngica de extractos etanólicos de dos morfotipos de Raphanus raphanistrum L. sobre tres hongos fitopatógenos.

Bioagro, 27(1),3-10. https://www.redalyc.org/pdf/857/85741584002.pdf

SAMSIDAR A, Siddiquee S, Shaarani MS. 2018. A review of extraction, analytical and advanced methods for determination of pesticides in environment and foodstuffs. Trends. Food. Sci. Technol. 71:188-201. https://doi.org/10.1016/j.tifs.2017.11.011

SEETHA J, Reddy R, Ramanjaneyulu R. 2010. Evaluation of certain plant extracts and antagonist against Fusarium solani and Alternaria tenuissima, the incitants od root rot and die-back diseases of Mulberry. Int. J. Indust. Entomol. 20:1-5. http://www.koreascience.or.kr/article/JAKO201019451499104.page

STASHENKO EE, Jaramillo BE, Martínez JR. 2003. Comparación de la composición química y de la actividad antioxidante in vitro de los metabolitos secundarios volátiles de plantas de la familia Verbenaceae. Rev. Acad. Colomb. Cienc. 27 (105): 579-597. ISSN 0370-3908. http://www.accefyn.com/revista/Vol_27/105/8-COMPARACION.pdf

TAMILSELVI N, Arumugam T. 2017. Breeding Approaches for Sustainable Vegetable Production-A Review. Int. J. Curr. Microbiol. App. 6:2845-2860. https://doi.org/10.20546/ijcmas.2017.611.336

TOUBA E, Zakaria M, Tahereh E. 2012. Anti-fungal activity of cold and hot water extracts of spices against fungal pathogens of Roselle (Hibiscus sabdariffa) in vitro. Microb. Pathog. 52:125-129. https://doi.org/10.1016/j.micpath.2011.11.001

VÁSQUEZ D, Montes R, Jiménez A, Flores HE. 2014. Aceites esenciales y extractos acuosos para el manejo in vitro de Fusarium oxysporum f. sp. lycopersici and $F$. solani. Rev. Mex. Fitopatol. 31:170-179.

http://www.scielo.org.mx/scielo.php?script=sci_arttext\&pid=S0185-33092013000200008 
WHITE TJ, Bruns T, Lee S, Taylor JW. 1990. Amplification and direct sequencing of fungal ribosomal RNA genes for phylogenetics. pp. 315-322 In: Innis, M. A.; Gelfand, D. H.; Sninsky, J. J.; White, T. J. (Eds.). PCR Protocols; A Guide to Methods and Applications, Academic Press, Inc., New York. http://blade1.uniquindio.edu.co/uniquindio/revistainvestigaciones/adjuntos/pdf/f4d9_n19 18.pdf

YOSSEN VE, Conles MY. 2016. Eficacia de fungicidas in vitro para el control de Fusarium oxysporum y $F$. proliferatum, agentes causales de marchitamiento en el cultivo de orégano en la Argentina. Revista industrial y agrícola de Tucumán, 91(1), 19-25. https://riat.eeaoc.org.ar/ojs/index.php/riat/article/view/v91n1a03/33

ZAKER M. 2014. Antifungal evaluation of some plant extracts in controlling Fusarium solani, the causal agent of potato dry rot in vitro and in vivo. Inter. J. Agri. Biosci. 3:190195. www.jjagbio.com

ZAMORA-NATERA F, García-López P, Ruíz-López M, Salcedo-Pérez E. 2008. Composición de alcaloides en semilla de Lupinus mexicanus (Fabaceae) y evaluación antifúngica y alelopática del extracto alcaloideo. Agrociencia. 42:185-192. http://www.scielo.org.mx/scielo.php?script=sci_abstract\&pid=S1405-

$31952008000200006 \&$ lng $=$ es\&nrm=iso 